\title{
La trilogie romanesque bretonne et maritime de Michel Mohrt
}

\section{Pascal Rannou}

\section{(e) OpenEdition}

1 Journals

\section{Édition électronique}

URL : http://journals.openedition.org/studifrancesi/8150

DOI : 10.4000/studifrancesi.8150

ISSN : 2421-5856

Éditeur

Rosenberg \& Sellier

\section{Édition imprimée}

Date de publication : 1 mai 2009

Pagination : 59-75

ISSN : 0039-2944

\section{Référence électronique}

Pascal Rannou, «La trilogie romanesque bretonne et maritime de Michel Mohrt », Studi Francesi [En ligne], 157 (LIII | I) | 2009, mis en ligne le 30 novembre 2015, consulté le 12 janvier 2021. URL : http:// journals.openedition.org/studifrancesi/8150; DOI : https://doi.org/10.4000/studifrancesi.8150

\section{(c) (i) $\odot$}

Studi Francesi è distribuita con Licenza Creative Commons Attribuzione - Non commerciale - Non opere derivate 4.0 Internazionale. 


\section{La trilogie romanesque bretonne et maritime de Michel Mohrt}

Né à Morlaix en 1914, Michel Mohrt n'est pas, loin s'en faut, l'écrivain breton de langue française le plus connu. Son élection à l'Académie française, en 1975, ne l'a pas rendu plus populaire dans une Bretagne que son œuvre n'a pourtant jamais cessé de célébrer. Est-ce l'éloignement d'un homme que sa vie a appelé à travailler dans la capitale depuis de longues années? C'était pourtant aussi le cas de Guillevic, c'est encore celui de Yann Queffélec, c'était celui de Jean Rouaud, avant son retour récent à Campbon. Peut-être est-ce parce que Michel Mohrt est trop catalogué politiquement? La Bretagne l'a elle-même longtemps été, mais pas, il est vrai, l'intelligentsia qui crée les modes et décerne les accessits. Chroniqueur au Figaro pendant longtemps, peutêtre Michel Mohrt a-t-il été victime du préjugé qui veut qu'un écrivain de droite ait forcément un style compassé, ampoulé... académique, en somme, ce que son élection au sein de la docte assemblée aurait contribué à entretenir!

Autant d'idées reçues que le présent article souhaite contribuer à rectifier, en présentant à ses lecteurs une trilogie romanesque que Michel Mohrt a consacrée à la Bretagne, à son histoire, à son peuple, et aussi à son paysage maritime: celui de la Manche, qu'il connaît bien. Il y a navigué sans relâche, sur les traces d'un glorieux «pays» auquel il n'a cessé aussi de rendre hommage: le poète Tristan Corbière, né comme lui à Morlaix. Cette trilogie comprend Le Serviteur fidèle, La Prison maritime et Les Moyens du bord. Le premier de ces romans a été publié chez Albin Michel, en 1953; les deux autres chez Gallimard, en 1961 et en 1975.

L'action du Serviteur fidèle ${ }^{1}$ se situe à Moguérou, lieu-dit imaginaire de la côte trégorroise. Annick et son frère Jean-Yves, tôt orphelins, ont été élevés par leurs grands-parents, aidés par deux domestiques, Rine et Lomic, qui est muet. A leurs jeux d'enfants participe aussi Louis, fils de la laveuse, qui tombe amoureux d'Annick. Mais ni Rine, ni Jean-Yves, ni la grand-mère ne voient d'un bon œil cette relation avec le fils d'une laveuse, un cancre qui plus est, et qui aime l'emmener en mer sur sa barque L'Aimée. Lomic, de plus, lui garde rancune: Louis s'est moqué de lui et de son handicap, naguère, avec les autres garnements du village. Un jour, pris par la marée, Louis et Annick doivent gagner un port voisin et y passer la nuit. Le lendemain, JeanYves vient chercher sa sœur en taxi. Celle-ci veut rentrer sur L'Aimée. Un brève lutte s'ensuit entre les deux hommes, qui tombent à l'eau, mais c'est Louis qui s'est montré le plus vaillant. Suite à cet esclandre, Louis est alors contraint de devenir «petit clerc» à Lannion, chez un cousin de Mlle Antoinette, la dame philanthrope qui lui payait jusque-là ses études. Cela l'éloigne d'Annick, qui fait, quelques années plus tard, la connaissance d'un groupe de jeunes étudiants, amis de son frère. Parmi eux émerge la personnalité de Pierre, cultivé, sportif, aux charmes de qui elle succombe, et qu'elle épouse. Jean-Yves n'assiste pas à leur mariage. Annick revoit Louis, et semble toujours éprouver des sentiments forts pour lui. 
La guerre survient. Jean-Yves est libéré d'un camp de prisonniers parce qu'il a promis d'occuper un poste important, à Vichy. Pierre meurt dès les premiers combats, Jean-Yves est de plus en plus compromis dans la collaboration. Louis, par contre, est devenu un des chefs de la Résistance, qui veut la peau de son ancien camarade. À la fin de la guerre, Jean-Yves regagne la Bretagne, et veut s'enfuir par mer avec l'aide de Lomic. Mais la bande de Louis le traque. Un échange de coups de feu a lieu. Un des résistants abat Jean-Yves, dont la mer rendra quelques jours plus tard le corps défiguré. C'est Lomic qui le découvrira. Les libérateurs occupent la propriété du Moguérou, coupent les arbres. Le grand-père meurt. Annick gagne Paris. Des années plus tard, elle revient, et retrouve Louis, à qui elle donne un rendez-vous amoureux dans son jardin, malgré la mort de Jean-Yves. Mais Lomic rencontre Louis, et le tue. Annick décide qu'il faut lancer son cadavre à la mer. Puis elle quitte Moguérou pour toujours.

Le Serviteur fidèle est une tragédie familiale dont émerge un vague relent d'inceste, vu le type de relation qui unit Annick et son frère. C'est une tragédie sociale aussi, puisque les personnages sont finalement victimes des préjugés de caste qui interdisent l'amour d'Annick et du prolétaire Louis.

L'intrigue de La Prison maritime est moins linéaire, plus touffue. L'auteur, il est vrai, tient ici à rendre hommage aux auteurs de romans d'aventures, et notamment de récits maritimes, comme Conrad, qu'il connaît bien. Rappelons que Michel Mohrt a, de plus, traduit certains romanciers du «Old deep South» américain, parmi lesquels William Styron et Robert Penn Warren.

L'action débute, cette fois-ci, en 1923. Le narrateur, Hervé, habite une propriété encore nommée Moguérou, en Lesguivy, localité imaginaire voisine de Saint-Yvinec, chef-lieu dont le nom est lui aussi fictif. Ses parents ayant disparu lors d'une promenade en mer, il a été confié au Bon-Pasteur, de Brest, où il suit les cours de l'abbé Guern, dit le Grand-Foc. Ce fin navigateur professe des convictions nationalistes bretonnes, teintées d'un panceltisme chrétien primitif à la fois fumeux et visionnaire. L'abbé lui fait un jour visiter la prison maritime de l'arsenal, où il note la présence d'un détenu nommé Lévêque, condamné pour faits d'autonomisme.

Le prestigieux cotre le Roi-Arthur mouille à Lesguivy. Son propriétaire, Olivier de Kersangar, cherche un matelot. Hervé propose ses services, et le voilà embarqué aux côtés du capitaine et de sa maîtresse, Lady Cécilia, sorte de vamp des mers, séductrice et nymphomane. Le Roi-Arthur doit récupérer en Irlande une marchandise inconnue du narrateur, avant de la livrer à il ne sait qui, en Bretagne. Le Grand Foc est de la combine, ainsi que deux militants bretons, Robert et Spagniol. La première sortie en mer est laborieuse: le mât casse, et l'équipage doit regagner Saint-Yvinec. Hervé y fait la connaissance du Président R..., du tribunal, et de ses trois filles, dont Iris, qui ne lui déplaît pas. La conversation, qu'animent les filles du Président, roule sur une affaire qui oppose de longue date l'Angleterre et la France, et concerne la propriété d'un archipel, nommé Beniget. De retour à bord, Hervé y rencontre Tugdual, cousin d'Olivier, qui fera désormais partie de l'équipage. Le président verrait bien en Hervé son futur gendre, qu'il pourrait nommer à un poste dans la magistrature locale. Mais, à la croisée des voies, le jeune matelot opte pour l'aventure et renonce à ces perspectives bourgeoises.

Lors d'un combat en mer, l'équipage du Roi Arthur combat les Anglais, auxquels il reprend un îlot de l'archipel des Beniget. Puis il gagne l'Irlande, où il retrouve Lévêque, rebaptisé Bishop, qui enseigne dans une université. Hervé y fait aussi la connaissance d'un curieux personnage, Arthur Saint-Arthur, érudit, ami de Gide, qui 
recommande au jeune homme la lecture de Toulet et de Segalen, et l'invite à perdre au plus vite son pucelage. Hervé y arrivera, dans les bras de lady Cécilia, après avoir failli céder aux charmes de Jennifer, une américaine de passage au physique plutôt viril. On retrouve Saint-Arthur à Ballinajoy, propriété du mari de Cécilia, qui s'avèrera n'être en fait que la «fille d'un épicier en gros de Saint-Brieuc»². L'équipage se retrouve à Londres, où Hervé participe au vol, à la Tate Gallery, un tableau d'inspiration celtique de Burne-Jones, afin de le rendre à l'Irlande. Hervé ne peut résister à l'envie d'acheter les services d'enfants prostituées, à Piccadilly Circus.

Le Roi-Arthur refait route vers la Bretagne, mais essuie une tempête et s'échoue sur la plage des sables d'or, où les douaniers arrêtent l'équipage, à l'exception de Tugdual, qui se sauve et se noie. Un douanier rancunier abat le chien Du, qui l'avait mordu au début de roman. Le bateau contenait des armes destinées au mouvement breton, ce qu'Hervé ignorait. Il est emprisonné à Pipipol, mais le Président R... l'en fera bientôt sortir. Olivier sera condamné à un an de prison, puis mourra pendant la guerre d'Espagne. On apprend, dans l'épilogue, qu'Hervé a vécu douze ans avec Cécilia, que Saint-Arthur est mort de froid, à Paris, pendant la deuxième guerre mondiale, que Spagniol et Bishop ont été exécutés à la Libération.

Les Moyens du bord n'offre pas d'intrigue réelle. Il s'agit plutôt de la chronique du village de Lesguivy, encore lui, peu avant le brasier de 1939. Cette chronique est toutefois vécue à travers le parcours de Guillaume Le Cormier, professeur de droit, spécialiste de l'histoire de Bretagne, issu d'une vieille famille bourgeoise, et dont la vieille mère vit toujours. Séparé de sa femme, il a un fils, Ronan, qui étudie le Droit, mais aurait voulu devenir peintre. Lesguivy est fréquenté par les «estivants de marque»: l'ambassadeur Jules Bataillard, le commandant Rouzic, Mlle Trébabu, Mme de Vergerennes, qui entretiennent dans cette station balnéaire un climat qui se veut mondain.

L'abbé Flohic, curé de la paroisse, venu du Sillon de Marc Sangnier, veut mettre un terme à des traditions qu'il juge païennes et folkloriques: cérémonie de la quenouille, à la messe; culte à la Vierge noire, bénédiction des bateaux, et accepte que l'on déplace le vieux cimetière qui entourait l'église. Il s'attirera l'animosité des fidèles bourgeois, qui le feront déplacer à Brest, dans un quartier «rouge», l'évêque étant plus tolérant envers les prêtres maurrassiens.

On assiste aux amours parallèles du père et du fils. Guillaume tombe amoureux de Grace, une vacancière américaine. Ronan a une liaison avec Martine de Parlieu, fille de l'ancienne maîtresse de son père, Hélène. Mais Martine lui préfèrera finalement Xavier Lenfant, un militant autonomiste fasciste, qui périra en mer en tentant de sauver son ami Ronan: ce dernier avait bravé la tempête par dépit amoureux. L'abbé Flohic, qui a assisté au naufrage du Coantik, le bateau des Le Cormier, secourt Ronan, sur le rivage, et le ramène au village. L'épreuve amènera le père et le fils à se réconcilier. Parmi les anecdotes qui secouent la chronique, mentionnons la mort de la mère de Guillaume, encornée par une vache lors d'un vente aux enchères; l'assassinat par Xavier d'un gars du village, membre d'une bande qui venait le chahuter; le dialogue de sourds entre Guillaume, qui veut restaurer une chapelle, et l'abbé Flohic, qui n'en voit pas l'intérêt, et préfèrerait que l'argent consacré à cette cause aille à des œuvres sociales.

(2) La Prison maritime, Paris, Gallimard (coll. Blanche), p. 218. 
Le Serviteur fidèle, La Prison maritime et Les Moyens du bord adoptent donc trois tonalités fort différentes. Le premier de ces ouvrages a la brièveté et l'épure d'une tragédie de Sophocle. Le second est un roman d'aventures maritimes qui se revendique comme tel, et qui en exploite plaisamment les ficelles. C'est le seul des trois récits dont les chapitres sont intitulés, selon la tradition du roman d'aventures. Et ces sous-titres sont évocateurs, qu'ils présentent un personnage («Le grand Foc», «Le chien Du»); un objet («Le cotre à tape-cul»); un événement («Le combat», «La danse au clair de lune», «La tempête») ou un lieu («Ballinajoy», «La plage des sables d'or»). Les chapitres s'ornent également d'épigraphes empruntées à Jules Verne, Defoe, Corbière, Chateaubriand, Shakespeare... modèles illustres (parmi d'autres, moins connus), qui suscitent un horizon d'attente: en si bonne compagnie, le narrateur ne saurait ennuyer son lecteur! Lady Cécilia rappelle les aventurières telles que la Milady, de Dumas. Rastignac, dans Le Père Goriot, se trouve à la croisée des chemins, entre la voie du crime que lui propose Vautrin, et celle de l'ascension par des moyens légaux et mondains. Il en est de même pour Hervé, qui tranche en faveur de l'aventure et éloigne la solution du mariage et de l'Establishment. La Prison maritime est donc un roman d'apprentissage pour un jeune matelot orphelin qui, parti de rien, découvre la mer et l'amour avant de devenir un narrateur plus rassis, spectateur ironique de sa propre existence. Les Moyens du bord est une chronique, a-t-on dit, et une méditation sur les vanités de l'existence, telle qu'elle se déroule devant un personnage principal déjà mûr. Mais c'est aussi un roman d'apprentissage, voire de formation, pour Ronan, qui franchit le cap initiatique de la tempête avant de retrouver sa vocation de peintre.

Les trois romans évoquent également l'initiation amoureuse de leurs personnages, ou la reprise de leur vie amoureuse, pour Guillaume. Ceux-ci sont ou orphelins (Hervé, Annick et son frère), ou éloignés de leurs parents (Ronan): les héros des romans d'aventures sont souvent débarrassés des contingences familiales. Plus libres, leur vie est une page blanche où ils auront tout à écrire, sans rien devoir à un atavisme aliénant. La trilogie convoque les mêmes lieux imaginaires: Lesguivy, Saint-Yvinec, Moguérou, qui ne sont pas habités par les mêmes héros. On y retrouve Mlle Trébabu, Tityves, Antoinette... qui désignent toutefois des personnages différents. Une Mme de Kersangar apparait ainsi dans Le serviteur fidèle (p. 17), et elle n'a rien à voir avec le commandant du Roi-Arthur. D'autres ressemblances surgissent: il est question, dans La Prison maritime (p. 223) et dans Les Moyens du bord (p. 91) du cimetière déplacé de Lesguivy. Dans ce dernier roman, la vente aux enchères fatale à Mme Le Cormier se tient dans un château, «construction dans le style néo-gothique de la fin du XIXème siècle, recoupé par le style normand des villas balnéaires»(p. 163). La résidence du président R... est, elle aussi, «une demeure prétentieuse, bâtie dans le style néo-gothique en faveur à la fin du siècle dernier, avec des tours crénelées et des fenêtres à meneaux et à vitraux de couleur»(p. 99). Le Moguérou héberge les héros des Moyens du bord comme ceux du Serviteur fidèle, qui ne s'y rencontrent pourtant pas. Marie du Bilou, bonne des Le Cormier, est le pendant de Rine, du Serviteur fidèle. C'est par de tels détails se remarque aussi la cohérence qui unit les trois romans. Mais cette cohérence est surtout visible dans l'importance que Michel Mohrt accorde à la Bretagne et à la mer.

(3) Nous renvoyons à l'édition Folio, parue en 1982. Nous désignerons parfois, pour gagner en lé- gèreté, les romans par leurs initiales: $S F, P M, M B$, et ferons suivre les références paginales. 
La présence de la Bretagne est, tout d'abord, sensible à travers celle de sa langue. Les toponymes celtiques impriment au texte une musicalité particulière: Kersauzon, Locmillau, Toul-ar-Christ, Plouvarneïz, Ploumildut, Lanevor, Pors-ar-Viliec, dans Le Serviteur fidèle; Toul-ar-Sauzon, Prat-ar-Coum, Ker Izel, Gaouïat, dans Les Moyens du bord ont un coefficient d'étrangeté qui doit rendre les lieux de l'action exotiques aux oreilles non bretonnes. Pour les autres, ces noms de lieu résonnent avec familiarité, même s'ils sont inventés: Locmillau, Ploumildut, Lanevor semblent des composés de Locmélar et Guimiliau; Ploumanac'h et Lanildut; Lanmeur et Plouvorn. Michel Mohrt forge des toponymes qui paraissent authentiques, contrairement à beaucoup d'écrivains non bretonnants qui écrivent n'importe quoi. Les anthroponymes convoquent des Lomic, Larvor, Scanff, Le Goff $(S F)$; des Coatcoz, de Kercoät, Trébabu, Kerzampar $(M B)$. N'oublions pas le chien Du et les cousins Kersangar $(P M)$ ainsi que le bateau Coantik ou «petit mignon» $(M B)$, dans le roman où l'on désigne Guillaume par son diminutif breton: Lomic (p. 33).

Mais la présence de la langue bretonne ne se réduit pas à celle de noms réels ou inventés. La trilogie de Michel Mohrt montre pour elle une véritable fascination, de la part de quelqu'un qui ne l'a jamais apprise. Plusieurs expressions non traduites parsèment ainsi ses récits. Il s'agit surtout d'interjections: «Gast!» («putain!» $\left.{ }^{4}\right), \ll F i$ dan Doué!» («Par la foi de mon Dieu!» ${ }^{5}$, «ma las Doué!» (《malheur à Dieu!»), ou de mots que l'auteur juge peut-être transparents à quiconque a une petite habitude de la Bretagne: «Mam» («mère»), «va Jésus!» («mon Jésus!»), voire «chupen et bragou braz» («veste et pantalon bouffant»), «penn baz» («bâton»), «paotr» (《garçon»), et aussi «carabassen» («bonne du curé»), «gouspérou» («vêpres»), «ya, kenavo» («oui, au revoir»), «O Breiz ma bro!» («O Bretagne, mon pays!»), «touquen» (femme portant la toukenn, coiffe de l'ancien évêché de Tréguier), «jabadao» (danse du Léon). Notons la graphie curieuse de «shuppen» (PM, p. 288) et, dans ce même roman, l'expression «Breiz Atao»(«Bretagne toujours!») que lance, au tribunal, Olivier de Kersangar (p. 333). Parfois, l'auteur francise le breton, comme le font les bretonnants de naissance. Il évoque ainsi «le corps (de Jean-Yves) rusê sur les rocher» (SF, p. 110). L'usage des italiques souligne, souvent, mais pas toujours, l'emprunt à une langue autre.

A quelques exceptions près, où figurent aussi des fragments de cantiques qu'il a pu lire dans des missels (SF, p. 45). Michel Mohrt écrit le breton phonétiquement. Il aurait fallu écrire: «Fid'am Doue, Mallozh Doue, Mamm, chupenn, karabassen, toukenn». Doit-on lui reprocher de ne pas avoir essayé d'apprendre à l'écrire correctement? C'est aussi le cas d'écrivains bien plus jeunes, comme Michel Le Bris $^{10}$. Il faut se replacer dans le contexte des années Trente, très hostile à la langue bretonne, où il a été en contact avec cette langue. Cela n'avait rien pour l'encourager à apprendre à l'écrire, ni à la parler pour un jeune homme dont la classe sociale n'était pas celle du menu peuple des bretonnants, et qui ne pouvait pas espérer l'utiliser dans sa vie professionnelle. Mais ce n'est pas cela qui a empêché Michel Mohrt d'illustrer, à quatorze ans, la pièce Gorsedd Digor, de Jakez Riou...

L'auteur consent souvent un effort de traduction: «"La paix, Du!" cria le capitaine à l'adresse du fauve dont j'appris ainsi le nom qui, en breton d'Armorique, signifie "noir"» $(P M$, p. 28). Cela confère à l'animal un prestige supplémentaire, que l'emploi du monosyllabe seul n'aurait pas suffi à véhiculer. Guillaume traduit aussi,

(4) $P M$, p. 90.
(5) Ibid., p. 108.
(6) PM, pp. 8 et 243.
(7) $S F$, pp. $7,14,49,59,106$.

(8) $M B$, pp. 69, 149, 152, 186, 190, 260.

(9) C'est à dire: traîné.

(10) Dans Un Hiver en Bretagne (Paris, NIL, 1996, réédité en coll. Points-Seuil) par exemple. 
avec tendresse, le nom du chat Fridu, ou «nez noir», dans Les Moyens du bord (p. 28). Le narrateur de La Prison maritime nous signale que «le Grand Foc (l') appelait souvent par la forme celtique de (s)on prénom, Hervé, dont le sens est "qui est apte au combat"». Cela annonce l'atmosphère épique où nous fera bientôt plonger l'action. Par deux fois, on lit que le mot «Saozon» ou «Saxons» veut dire «Anglais», en breton ${ }^{11}$. C'est, chaque fois, amené par une digression sur l'histoire ancienne de la Bretagne. L'information étymologique authentifie l'anecdote. Celle qui concerne l'île Couëron: «Coët: bois, ronn: fragment» (PM, p. 105) légitime l'assaut de l'équipage du Roi Arthur sur l'archipel des Beniget (ou «béni»), Breton de nom, donc Français aussi. Il est, cela dit, peu probable qu'un îlot proche des Minquiers ait un nom aussi breton. On sait que Beniget est un îlot proche d'Ouessant: Michel Mohrt réinvestit la toponymie et la met au service de sa fiction.

En Irlande, Tugdual s'adresse à un voisin de bar en breton, espérant en vain se faire comprendre: «mad eo, tom eo», dit-il, ce que le narrateur traduit: «C'est bon, c'est chaud»(PM, p. 152). L'anecdote est savoureuse, et montre la naïveté d'un militant breton dont le panceltisme se heurte aux barrières linguistiques. Une scène similaire fait, dans Les Moyens du bord, se rencontrer Xavier et un pilier de bar anglais: tous deux sont vite «un peu saouls tous les deux, Xavier et lui. Drunk, meo. Bière et whisky» (p. 139). Traduire en deux langues le mot «saoul» ajoute à la truculence de la scène. C'est à cet effet que vise un épisode du Serviteur fidèle, où Rine nous montre les grands-parents qui se chamaillent:

C'est tous les jours la même comédie: «Où vas-tu, à cette heure-ci? Tu vas encore à Gouspérou? Je te préviens que je me mets à table». Il appelle toutes les prières et les offices: Gouspérou, alors qu'il sait très bien que c'est seulement les vêpres qui se disent Gouspérou, en breton. Mais il aime à se moquer un peu des prières de ta Mam et de tous les bulletins des missions étrangères qu'elle reçoit... (p. 13).

Il arrive que le vocable non traduit fasse sourdre une atmosphère fantastique: «Les poulies se mirent à geindre comme des âmes tourmentées par l'Ankou», confie ainsi le narrateur de La Prison maritime (p. 267). Mais quand celui des Moyens du bord évoque «la fin de septembre, le mois de "la paille blanche"» (p. 265), sans dire que cela traduit le breton «gwengolo», il risque de dérouter le non-bretonnant, tout en le charmant par une touche de poésie moins frappante en breton, où le mot est courant. La syntaxe des bretonnants apparaît aussi dans les dialogues, où ils emploient systématiquement le pronom démonstratif en lieu et place du simple pronom personnel: «Celui-là est détraqué» (SF, p. 15), «Celui-là va en Angleterre» (PM, p. 16). Beaucoup se sont moqués naguère de cette syntaxe jugée «plouc»: Michel Mohrt la restitue sans nulle intention de ce genre, simplement par souci d'authenticité. C'est ainsi que le narrateur des Moyens du bord exprime son profond respect pour «les syllabes rauques de la langue celtique» (p. 115). «Sur notre passage, les conversations tenues dans cette langue âpre que j'étais si mortifié, moi un autonomiste, de ne pas comprendre, s'arrêtaient», confie Hervé (PM, p. 289). Nul doute que l'auteur s'exprime derrière ses personnages, comme il le fait encore dans ce cri du cœur: «ma grand-mère parlait breton et moi je ne sais dire que Tamm' bara mar plij et D'ar gwele ma jolie... $\gg^{12}$. Michel Mohrt est sans doute l'un des écrivains bretons de langue française qui puise le plus au vivier lexical d'une langue palimpseste dont il fait un

(11) PM, p. $133 ; M B$, p. 205.

(12) C'est à dire: «Un bout de pain, s'il vous

plaît» et «Au lit, ma jolie», MB, p. 206. 
personnage de ses récits. Mais l'hommage qu'il y rend à son pays natal se signale aussi dans la présence marquée de son Histoire.

La chronologie des trois romans n'épouse pas celle de leur fiction. Publié en 1953, Le Serviteur fidèle se déroule dans les années 1930 et atteint son point culminant avec l'exécution de Jean-Yves, à la Libération. La Prison maritime (1961) se déroule en 1923, comme on l'apprend dès la première ligne. Mais les prolepses propres au récit-cadre d'un narrateur qui a vécu les faits qu'il raconte nous projettent jusqu'à la deuxième guerre mondiale. Les Moyens du bord (1975), enfin, se situe au moment de la guerre d'Espagne: les bigotes de Lesguivy s'indignent, en effet, du sort que les Républicains font à la religion catholique. La mère de Guillaume, par contre, épouse la cause opposée, et ce malgré ses convictions religieuses: «Je suis pour la Catalogne libre, dit la vieille dame. La Catalogne doit être libre, comme la Bretagne devrait l'être» (p. 50).

Cette trilogie se déroule donc à une époque sensible et douloureuse de l'Histoire de la Bretagne: celle où le mouvement breton était clairement ancré à l'extrême droite, et baignait dans une idéologie confuse et dangereuse. C'est bien sûr Le Grand Foc, «un des théoriciens les plus écoutés du mouvement autonomiste en Bretagne» (PM, p. 38), qui illustre le mieux cet état d'esprit. «Qu'on n'attende pas de moi un exposé de la doctrine politique de l'autonomisme breton», écrit Hervé (p. 39): pure prétérition, puisque c'est ce qu'il va faire trois pages durant, et tout d'abord en invoquant les raisons qui incitent certains à devenir autonomistes: dépérissement économique, oppression linguistique; érection, à Rennes, d'un monument humiliant représentant la Bretagne agenouillée devant la France ${ }^{13}$, exil parisien engendrant misère et prostitution (pp. 38-39). Mais le Grand Foc a une vision plus romantique de sa cause: «l'abbé ne concevait l'autonomisme breton que dans l'ensemble de la Renaissance celtique» (p. 39). Hervé nous cite alors les maittres à penser de son mentor: Ossian, Walter Scott, La Villemarqué; mais aussi les écrivains et hommes politiques irlandais: Douglas Hyde, Synge, Yeats:

Sans ce magnifique essor culturel, l'Irlande n'aurait jamais conquis son indépendance. Le Grand Foc rêvait d'une confédération qui eût rassemblé les morceaux épars de l'ancienne famille celtique [...]. Le gaëlique et le brittonique, les deux familles des langues celtiques toujours en usage [...] assureraient l'unité culturelle et linguistique de l'ensemble [...] Dans sa confédération celtique, l'abbé voyait le dernier bastion de l'Occident chrétien. Combattu de toutes parts, celui-ci se repliait sur ces côtes sauvages, ces îles et ces îlots rocheux où le christianisme avait gardé ses vertus primitives [...]. C'était de là qu'il faudrait partir à la reconquête du monde occidental comme, au Vème et au Vème siècle, les moines d'Irlande et du pays de Galles étaient partis pour évangéliser l'Europe redevenue barbare [...]. Alors, lavée de ses péchés et de ses erreurs dans un bain de sang, l'Occident retrouverait la tradition perdue de l'âge d'or (p. 40).

Hervé puise à ces idées sans retenue, notamment devant Iris, qui lui oppose «le nombre ridicule des partisans de l'indépendance bretonne», ce à quoi son interlocuteur rétorque par l'argument des «minorités agissantes» (p. 119), après avoir fait l'éloge d'Eamon de Valera, le président irlandais dont le Grand Foc lui avait parlé. L'abbé illustre bien une certaine tendance du mouvement breton. On pense à

(13) Ce monument fut, en 1932, l'objet du premier attentat nationaliste breton. 
l'abbé Perrot, bien sûr, même si le mentor d'Hervé n'a pas été, comme Perrot, abattu par la Résistance. En fait, nous dira Hervé, «le grand Foc était un intellectuel prudent et douillet, soucieux de ses aises: il préféra un exil confortable dans un pays ami et un poste de français dans un collège du Kent aux ennuis qui l'attendaient dans son diocèse» (p. 333). Par contre, ses disciples Spagniol et Lévêque, eux, furent «tués tous deux par des maquisards» (ibid.). Lévêque enseignait auparavant dans une université irlandaise, comme Roparzh Hemon, l'écrivain bretonnant poursuivi après la guerre. Michel Mohrt semble se souvenir de ces personnages réels, et en nourrir sa fiction. L'expression «Breiz Atao», que lance Olivier de Kersangar à son procès (p. 332), évoque bien entendu le parti nationaliste breton créé par Mordrel et Debauvais.

Xavier Lenfant est, lui aussi, un militant nationaliste. Mais il appartient à une ligue d'extrême droite, le Front patriotique, qui fomente des émeutes ailleurs dans l'Hexagone ( $M B$, pp. 43-44), et pas à un parti breton. Il expose assez confusément ses convictions devant un marin anglais rencontré à Saint-Hélier:

Sans doute, le mouvement autonomiste n'est pas très important, mais il existe, il exprime des aspirations éparses qui ne demandent qu'à... Des siècles de colonisation laissent des rancœurs ineffaçables [...]. Un peuple ne perd pas son identité tant qu'il conserve sa langue. On a tout fait pour le déposséder de celle-ci: les enfants punis dans les écoles, s'ils sont en train de parler breton, etc. Lui, Xavier, n'est pas pour une indépendance qui, aujourd'hui, n'est-ce pas, à l'heure, n'est-ce pas, où les nations tendent vers l'unité... dans son mouvement, il défend une régionalisation, une décentralisation, une large autonomie des provinces. Mais son mouvement, est, était unitaire. Il faudra bien que les provinces... Mon mouvement, le front patriotique, aujourd'hui dissous. Mais il renaittra, sous une forme ou une autre, il correspond à une nécessité politique, géographique, économique... (p. 134).

Le Front patriotique est un mouvement imaginaire. Pourquoi Michel Mohrt a-til inventé un parti, alors qu'il reprend le nom, authentique, de Breiz Atao? Peut-être par ce que ce dernier est entré dans la langue courante, après 1945, pour désigner un indépendantiste breton collaborateur. Xavier est aussi une figure romantique: excellent navigateur, engagé volontaire à dix-sept ans, il périra, on le sait, en voulant sauver Ronan. Il avoue sans ambages son admiration pour Hitler (p. 43). Xavier est à Ronan ce que le Grand Foc est à Hervé: un initiateur politique, un tentateur, un maître qui, de plus, lui ravit sa compagne. Jean-Yves n'est qu'un fonctionnaire que Vichy a recruté après l'avoir libéré d'un stalag $(S F$, p. 75). Ce n'est pas, non plus, un «Breiz Atao», mais son choix politique signera son arrêt de mort. Le Grand Foc est, par ailleurs, l'antithèse de l'abbé Flohic, qui ne cesse de fustiger les traditions bretonnes:

Il faudra bien, un jour, en arriver à supprimer processions et pardons en l'honneur de saints qui n'ont existé que dans l'imagination populaire - ces saints venus d'Irlande «dans des auges de pierre», dit la légende, comme si une auge de pierre pouvait flotter! (MB, p. 92).

Pour quelqu'un qui a forcément baigné dans le surnaturel chrétien, l'abbé Flohic paraît singulièrement rationaliste! L'histoire de la Bretagne est aussi présente, de manière moins agressive, dans Les Moyens du bord. Une rêverie sur le château de Tonquédec suscite une vision qui fait surgir le Moyen-Age, avec ses manants, ses «mercenaires» et ses «reîtres», ses «Saozon» aussi, «les Anglais, que l'on appelait les Saxons car on avait gardé la mémoire des pirates saxons et normands», dont un «garçon de ferme» annonce la venue de village en village (p. 204-208). Guillaume projette d'écrire une pièce sur Gilles de Bretagne, le frère assassiné du Duc François II. Il en expose l'argument devant Grace (pp. 196 à 201). Un voyage à Tréguier est l'occasion d'une autre rêverie, consacrée aux figures antinomiques de Saint-Yves et de 
Renan (pp. 191-192). Les grands noms de l'Histoire bretonne suscitent un accès de nostalgie désenchantée: «Cette Bretagne est morte comme l'autre, celle de Tréguier, comme celle de la chouannerie, il n'en reste que des ruines» (p. 206). Chez Hervé, par contre, l'évocation de «Warok et Hoël le Grand, Alain Barbe-Torte, Jeanne de Flandres, Jean de Montfort, Pontcallec...» éveille l'enthousiasme (PM, p. 42). Les grandes figures de l'histoire ducale: «les marches et les contre-marches de Simon de Montfort et de Charles de Blois, le connétable de Clisson, Beaumanoir au combat des Trente, appuyé contre un chêne comme un scarabée d'acier géant écrasé sur le sol, et buvant son sang» alimentent aussi la rêverie d'Annick (SF, p. 63).

Il serait intéressant de savoir si Michel Mohrt partage les idées qu'il attribue à ses personnages. Il est probable, vu la récurrence de ce thème, qu'il considère la cause bretonne avec une certaine sympathie. Les seuls propos anti-bretons de La Prison maritime sont tenus, lors de la réception finale chez le Président R... par un notable ivre que le narrateur caricature (pp. 312-313). Mais le parcours de ses «héros» et les commentaires qui les accompagnent montrent clairement qu'il condamne les extrémismes mis en scène. Décrivant les arguments de ses anciens camarades, Hervé s'en désolidarise avec force, longtemps après ( $P M$, p. 39). Le portrait final du Grand Foc, sagement réfugié en Angleterre, alors que ses disciples sont morts pour sa cause, est, on l'a vu, très sarcastique. Evoquant des perspectives de vie future avec Jennifer, Hervé englobe satire du mariage et de la mythologie irlandaise: «J'aurais fait quatre fils à ma princesse irlandaise, quatre géants blonds à qui nous aurions donné des prénoms de héros et de dieux: Diarmuid, Strongbow, Cuchullain...» (p. 258). Il brocarde ailleurs «l'anarchie celtique»(p. 68). Il souligne l'étroitesse d'esprit d'Olivier de Kersangar: «il semblait qu'il existât pour lui deux catégories d'individus: ceux qui appartenaient à la noblesse bretonne, et les autres» (p. 98) et met en doute sa sincérité: «Je n'ai jamais cru en la solidité de ses convictions. S'il embrassa la cause autonomiste, ce fût par goût de l'aventure» (p. 67). Lorsque l'équipage du Roi-Artbur reprend Beniget aux Anglais, il y fixe un pavillon français, non «le pavillon blanc semé d'hermines» (p. 138), les hommes de Kersangar ne partageant pas ses convictions. Hervé ne s'épargne pas lui-même: «L'histoire était le piège qu'il nous tendait: nous la sollicitions dans le sens de nos préjugés» (p. 41); «Même, je ne suis pas sûr que le fait d'avoir été autonomiste breton constitue une bien grande originalité» (p. 126); «Je m'étonne d'une fatalité qui me poussait à embrasser toutes les causes perdues de l'bistoire» (p. 221). L'humour même déconsidère la cause bretonne. L'équipage du Roi-Arthur vient d'aborder l'Irlande avec ses illusions. Les voici dans un pub où Tugdual s'efforce en vain de parler breton à son voisin:

«D'où venez-vous?» demanda l'Irlandais (...).

«De Bretagne», répondit Tugdual.

Si nous avions cru par cette déclaration provoquer un enthousiasme général, nous aurions été désappointés.

«Ah! Vous êtes français», dit l'autre.

Le plus simple fut d'en convenir (p. 152).

Rappelons aussi que les militants bretons ont, curieusement, des patronymes français: Lenfant, Lévêque, ou fréquents en Bretagne mais d'origine extérieure: Spagniol. L'auteur veut-il montrer par là que ce ne sont pas des Bretons authentiques? Le nom de Xavier Lenfant souligne-t-il la puérilité de son entreprise? Celui de Lévêque montre-t-il qu'on entre dans ce mouvement comme on entre en religion, avec l'abbé Guern, et avec les œillères que supposent un tel engagement? «Un chroniqueur d'extrême gauche» a écrit que Lenfant «menait ses fidèles "en bateau", allusion aux prouesses bien connues du yachtman» (MB, p. 44). C'est une boutade, 
mais peut-être cache-t-elle la pensée de l'auteur, quand on sait que les héros de $L a$ Prison maritime, ainsi que Jean-Yves, qui fuit en barque les résistants qui veulent sa peau, sont de redoutables navigateurs.

L'autonomisme breton décrit dans La Prison maritime et Les Moyens du bord est plutôt un nationalisme fascisant. Il ne semble pas qu'on ait pu concevoir, à l'époque, un autonomisme de gauche. La gauche, que représente l'abbé Flohic, est farouchement jacobine et opposée au régionalisme. À lire Michel Mohrt, on comprend mieux le discrédit jeté sur tout le mouvement breton après 1945 .

Notre trilogie fait également la part belle à la petite histoire et à la civilisation. La Prison maritime emprunte son titre à la prison de l'arsenal, haut lieu de l'histoire sociale et militaire de Brest. La mendicité est un phénomène sociologique qui n'a échappé à aucun observateur, dans la Bretagne du XIXème et du début du Xxème siècle. Michel Mohrt décrit, dans Les Moyens du bord, une mendiante pathétique nommée Julienne, qui fouille les rochers, son haveneau à la main, pour échanger ses crevettes contre un peu d'argent et un verre de rhum (pp. 149-152). Il évoque aussi ce déserteur qui fuit la mobilisation en 1914, et regagne le domicile maternel dans une malle, avant d'être dénoncé à la police, ce qui plonge sa mère dans la folie (pp. 147149). Cette anecdote ébauche un parcours narratif qui, développé, aurait pu donner matière à une nouvelle. Il est question dans La Prison maritime des valets de ferme qui, dans leur contrat, exigent «qu'il ne leur serait pas servi de saumon au repas plus de trois fois par semaine» (p. 295). Ce fait authentique est bien connu des historiens. De plus, tout comme on connaît les Sophistes grâce à Platon, leur contempteur, on a un bel aperçu des traditions bretonnes grâce à celui qui veut les vaincre: l'abbé Flohic. La plus intéressante est celle de la quenouille:

À la fin de la grand-messe du dimanche, les deux hommes qui avaient accompagné les quêteuses et étaient, comme elle, désignés pour l'année entière, saisissaient des quenouilles de lin fixées au sommet de hampes enrubannées et couronnées d'un bouquet de fleurs. Ils allaient saluer deux membres de l'assistance, repérés au cours de la quête, en inclinant devant eux les quenouilles. C'était là un honneur. Celui qui en était l'objet devait faire parvenir au presbytère une obole spéciale (p. 25).

On a déjà évoqué les autres: bénédiction des bateaux, culte à la Vierge noire, cimetière autour de l'église, missionnaires sur leur auge de pierre. N'oublions pas les saints bretons, «ces saints joufflus aux yeux ronds, étonnés de ce qui leur arrive (...) Quirec, Harent, Efflam...» (p. 285) qui ornent la chapelle Sainte-Barbe, où peint Ronan. Tout cela renvoie à un christianisme archaïque dont les manifestations sont, au moment où se situe le récit, d'autant plus précieuses qu'elles sont vouées à disparaître. Les allusions à deux motifs célèbres du légendaire breton amplifient la force évocatrice des deux tempêtes décrites dans La Prison maritime:

Ainsi, quinze siècles plus tôt, à ce même endroit de la mer où fuyait le Roi-Arthur, sur ce qui était alors une lande sauvage, un cavalier de la nuit portant en croupe sa fille galopait devant le raz de marée déclenché par la colère divine contre les habitants de sa capitale, l'Ys mystérieuse dont les tours s'aperçoivent par temps clair à marée basse et que certains situent au large de la baie de Douarnenez, d'autres de celle de Saint-Michel-en-Grève (p. 90);

Le grésillement de la pluie et le tambourinage des grêlons sur le pont, mêlés au fracas des vagues qui se brisaient sur le bordé faisaient une sarabande de tous les diables, sur laquelle se détacha un sifflement accouru du fond des ténèbres naissantes. Il passa au-dessus de moi, ter- 
rible, et dans les hauts du navire les poulies se mirent à geindre comme des âmes tourmentées par l'Ankou (p. 267).

L'auteur fait ici confiance aux compétences culturelles d'un lecteur qui saura identifier la célèbre allégorie de la faucheuse. Mais cela ne l'empêche pas de critiquer «la chienlit sophistiquée des biniousards du dimanche» (SF, p. 62), le vernis du folklore, qu'il oppose, un peu durement peut-être, à l'authenticité des traditions religieuses.

Deux des titres de nos trois romans renvoient à la mer. La Prison maritime renvoie au lieu où le Grand Foc officie en qualité d'aumônier. L'auteur n'évoque que très peu cet endroit, des pp. 53 à 59 de son long roman seulement, un peu comme Stendhal, qui ne mentionne la «chartreuse de Parme» éponyme de son livre qu'à la toute dernière page. Mais la prison est, pour Hervé, lieu d'un rituel initiatique. Il y découvre le monde inconnu des forçats, y aperçoit Lévêque, y découvre une mythologie qui transforme les prisonniers en héros:

Trois forçats, condamnés à mort, pouvaient se porter volontaires pour faire sauter la dernière poutre retenant la coque d'un navire qui s'apprêtait à glisser sur les rails enduits de tonnes de suif, avant d'aller danser dans le bassin où il serait achevé: si les malheureux parvenaient à éviter l'énorme masse qui, libérée, fonçait sur eux, ils avaient la vie sauve, mais le plus souvent ils périssaient écrasés (p. 53).

Le titre Les Moyens du bord renvoie, au premier degré, à la débrouillardise dont on doit faire preuve en mer, où l'on ne peut compter avec tous les services dont on dispose à terre. Au second degré, il désigne une façon de mener son existence tant bien que mal, à hue et à dia. C'est le cas de Guillaume, intellectuel doué mais trop dilettante, qui ne sait pas parler à son fils, n'arrive pas à retenir Grace, et ne renoue avec Ronan qu'après le naufrage qui a failli l'emporter. La tempête est donc, bien sûr, un rite de passage dans une vie de marin, et plus largement, une vie d'homme. Deux chapitres de La Prison maritime la prennent pour objet. Dans «La nuit des charpentiers», l'équipage du Roi-Artbur essuie un grain, dès la première sortie d'Hervé. La traversée commence calmement, mais des prémices fâcheuses surviennent:

Vers le soir du deuxième jour, le vent fraîchit.

Vous voyez, dit Auguste qui s'était instauré mon mentor et avait commencé à m'apprendre à faire des épissures «je n'aime pas ces vagues longues, ni cette écume, par exemple... Et je n'aime pas ce ciel» (p. 87).

\section{L'initiateur d'Hervé a pressenti la tempête dont la description s'ensuit:}

Alors, la voile se gonflait, les écoutes se tendaient en vibrant, éparpillant des gouttelettes d'eau de mer, la mâture entière ahanait sous la force du vent, et il semblait à chaque nouvel effort qu'elle fût sur le point d'être arrachée; l'amarre du canot, disparu dans une vallée profonde, battait la surface de l'eau rendue mystérieusement lisse et comme huileuse dans un demi-cercle au-delà duquel se devinait la muraille menaçante, prête à se fracasser et à nous engloutir sous l'avalanche de ses moellons et de ses parpaings, jetée bas par les mille trompettes du vent qui, plus de sept fois et de septante fois sept fois, en avaient fait le tour (p. 90). 
Les hyperboles, les métaphores, les personnifications d'éléments auxquels on attribue une volonté de nuire, le rythme houleux de la phrase périodique, l'allusion mythologique finale; tout cela concourt à la naissance d'une tonalité épique saisissante. C'est aussi le cas dans le chapitre intitulé «La tempête». Mais le narrateur a ici une intention pédagogique, qui élimine l'accusation de redondance:

Ayant déjà sacrifié au genre, je ne jugerais pas nécessaire de décrire cette nouvelle tempête, si je ne croyais utile d'indiquer au lecteur quelque peu familier avec la navigation à voile, les moyens que nous employâmes pour la combattre (...) (p. 267).

De fait, si le registre épique demeure, on voit ici comment l'équipage décide de «mettre à la cape», c'est à dire «toute la voilure amenée, de mettre le navire dans le lit du vent de façon de présenter à la mer le minimum de surface de la coque, au moyen d'une ancre flottante» (p. 268); puis de hisser un «tourmentin, petite voile de l'avant à parer par gros temps» (p. 269). Mais tout cela échoue, et l'on voit alors Tugdual «s'amarrer à bâbord au bas-hauban, près du ridoir» puis «s'élever avec lenteur, escaladant les enfléchures, menacés par l'espar qui oscillait au bout des drisses et autour duquel il essayait de passer un bout pour le tirer à lui et l'amarrer au hauban» (p. 272).

Les romans maritimes de Michel Mohrt sont aussi des romans qui s'adressent aux marins: le vocabulaire technique y est important, et, en l'absence de notes, le lecteur ignare est renvoyé au dictionnaire, ou à la perplexité. Le narrateur de La Prison maritime s'en justifie

Je me suis donné la preuve que je n'avais pas oublié les termes de navigation et étais encore capable de manœuvrer sur le papier, à défaut de manœuvrer sur la mer. Si je n'écrivais d'abord pour moi, peut-être devrais-je supprimer quelques expressions techniques qui risquent de rebuter le lecteur. Mais comment raconter une navigation en termes de terriens? Et où serait mon plaisir? (p. 37).

Ce roman a parfois l'allure d'un cours de navigation où l'on apprendrait à jongler avec les «winches à manivelles; chemins de fer à coulisseaux; bômes à rouleaux» (p. 63); à reconnaître «le mât de tape-cul légèrement incliné vers la poupe, l'immense bout-dehors qui lui sortait droit du corps maintenu par la sous-barbe, la barre du gouvernail passant au-dessus du banc de quart» du cotre Roi-Arthur (p. 13); à lire son parcours dans «les chariots de la Grande et de la Petite Ourse, Orion, Cassiopée, Bételgeuse, l'étoile du berger» (p. 82); mais aussi à manger du hareng et à boire du «vermouth Noilly-Prat» au «boujaron», avec les membres de l'équipage (p. 81)... Le lecteur initié apprécie, car il est en terrain de connaissance. L'ignorant de bonne volonté doit chercher à comprendre, mais l'abondance des technolectes ne nuit pas à la compréhension d'ensemble ni à l'intérêt porté au destin des personnages.

Dans Les Moyens du bord, la tempête est décrite différemment:

La grand-voile s'est déchirée.

Quatre tours de rouleaux! Quatre tours de rouleaux!

La drisse a pété la voile et s'est fendue, elle reste fixée à la bôme et s'effiloche dans le vent.

Ce pan de toile blanche qui traîne dans l'eau à bâbord! Impossible de l'enlever.

Mais qu'est-ce qui lui a pris?

Le foc tient. Il peut encore manœuvrer.

Le gouvernail tient. Ronan l'entend qui joue et fait entendre son bruit sourd, bois contre bois. Il a réussi à border l'écoute de grand-voile, à ramener la bôme qui foutait le camp.

Mais qu'est-ce qui lui a pris?... (p. 241). 
Le registre est moins oratoire, moins épique. Aller à la ligne si souvent crée un suspense angoissant. Le langage, plus courant, nous donne l'impression de lire les pensées de Ronan, comme le montre la dernière phrase, au discours indirect libre. Ici aussi, le personnage affronte la tempête comme un rite de passage, une épreuve qu'il s'impose après avoir été trahi par Xavier, son meilleur ami. C'est encore le cas dans Le Serviteur fidèle. Louis promène Annick sur sa barque. Mais il s'est endormi, et son amie a gardé le cap en rêvassant, sans penser à virer de bord. Quand Louis se réveille, «la côte n'était plus qu'une mince bande bleuâtre, et déjà, au couchant, une masse de brouillard se formait, qui allait fondre sur eux» (p. 43). Les deux passagers devront donc passer la nuit sur la côte opposée, au grand scandale de la famille d'Annick, mais pas à celui de la jeune femme: «Et il ne lui déplaisait pas de se compromettre avec Louis: quelque chose d'important, de décisif, sortirait peut-être de cette escapade» (p. 44). La transgression maritime a ici une valeur sentimentale, voire sexuelle. Dans les deux autres romans, il s'agissait de se mesurer aux éléments, de côtoyer la mort, de manière quasi suicidaire, pour Ronan. La mer fait s'enlacer Eros et Thanatos. Elle entérine, d'ailleurs, la sanction de la mort: c'est elle qui rend les corps de JeanYves, abattu au terme d'une lutte dont le décor est la mer (SF, p. 108), de Tugdual (PM, p. 315) et de Xavier (MB, p. 262). Les derniers moments de Tugdual, en lutte avec la barre, alors qu'il essaie de ramener le canot sur le rivage, sont d'une intensité dramatique remarquable.

Mais la mer n'est pas que violence. Michel Mohrt nous offre souvent, dans Les Moyens du bord, des tableaux plus sereins, des paysages qui sont de vraies marines:

Des voiliers sillonnent la baie en tous sens. Il souffle un faible vent de nordet et les bateaux avancent à peine. Les voiles blanches se reflètent dans une eau d'un bleu violet où les courants mettent des traînées émeraude. La côte s'estompe derrière une brume de chaleur. Deux voiliers, bord à bord, filent au plus près ( p. 65).

Une scène de genre, à la plage, rappelle au narrateur les tableaux de Boudin (p. 154). On apprend que la voilier de Marin Marie se trouvait, il y a peu, à SaintHélier (p. 139): ce navigateur en solitaire fut aussi un aquarelliste apprécié. Une scène de pêche à bord engendre une action vive et plaisante (p. 127). Grace et Guillaume mangent des fruits de mer. La description de cette scène est si riche en notations sensorielles qu'elle met l'eau à la bouche (p. 183). La bénédiction des bateaux évoque un tableau de Lemordant ou de Méheut. Michel Mohrt entonne ici un hymne aux bateaux rutilants et au petit peuple en costume chargé de dévotion; une symphonie allègre de couleurs, de mouvements et de bruits, chargée de tendresse et d'empathie pour cet univers humain humble et fort à la fois:

Les trois mâts, les mâts de flèches, le bout-dehors étaient reliés entre eux et à la coque par l'entrecroisement aérien des drisses, des haubans, des bas-haubans, des échelles de corde; et des canots de sauvetage, arrimés sur le pont, étaient suspendus aux bossoirs. A sec de toile, le navire portrait son grand pavois et les pavillons claquaient au vent. (...) A cinq pas derrière la frégate, quatre femmes dans leur costume de cérémonie, la grande cornette de dentelle gaufrée aux ailes qui frémissaient à chaque mouvement de tête, la pointe du châle noir à franges leur battant les chevilles, portaient la statuette d'argent de la Vierge (...) (p. 113).

La Prison maritime et Les Moyens du bord sont l'occasion de périples initiatiques en Irlande pour l'un, et à Jersey dans l'autre. Hervé et Xavier y rencontrent chacun un interlocuteur attentif et truculent: Arthur Saint-Arthur, et un certain Trelawney, dont le nom est un clin d'œil à L'Île au trésor. L'importance de la mer véhicule aussi une intertextualité récurrente: celle des Amours jaunes, de Tristan Corbière. 
On l'a dit, Corbière et Michel Mohrt sont tous deux morlaisiens, et tous deux ont navigué sans relâche sur la Manche. Mohrt voue une admiration totale au poète, et ne perd jamais une occasion de la manifester. C'est donc surtout le Corbière marin, celui de la section «Gens de mer» des Amours jaunes, qui apparaît dans notre trilogie. La seule exception à cela est la citation in-extenso d'un des «Rondels pour après», dernière section du recueil, que Guillaume récite à Grace ( $M B$, p. 157), sans que le nom du poète soit cité: voilà qui embarrasserait le lecteur qui ne connaît pas Corbière... mais qui dessine une complicité entre ceux qui le connaissent, l'auteur et son personnage! C'est là un cas de figure étonnant, dans l'arsenal des possibilités citationnelles. Il devrait s'agir, comme le dit Daniel Compère dans sa thèse sur Jules Verne, d'une copie, puisque «le texte emprunté est reproduit sans marque d'origine». Mais «l'emprunt reste identifiable» et tient donc aussi du «montage» ${ }^{14}$. Un phénomène similaire survient dans La Prison maritime. Hervé souhaite nous faire «comprendre le mépris du marin envers ceux que le poète appelle des “terriens parvenus"» (p. 79). Ces deux mots achèvent le poème de Corbière intitulé La Fin ${ }^{15}$, pastiche d'Oceano Nox de Hugo. Ils achèvent d'ailleurs aussi la section «Gens de mer». Là encore, l'allusion au «poète» est réservée aux «happy few»: en 1961, ils n'étaient pas si nombreux à connaître Corbière!

«Le phare de Corbière se dresse sur une pointe rocheuse. Au-delà, c'est la pleine mer» lit-on dans Les Moyens du bord (p. 141). C'est une allusion au poème intitulé Le Phare, allégorie phallique assez explicite ${ }^{16}$. Le profane pourrait prendre ce nom pour un toponyme. L'épigraphe de «La nuit des charpentiers», sixième chapitre de La prison maritime: «Voyez comme déjà l'apprentissage est dur», est par contre clairement attribué à son auteur, même si Michel Mohrt ne cite pas le poème dont ces mots sont extraits, en l'occurrence Lettre du Mexique ${ }^{17}$. L'épigraphe se situant horstexte, il s'agit donc plutôt ici, toujours selon les critères énumérés par D. Compère, d'un «renvoi explicite, ou référence» ${ }^{18}$.

On peut relever un bref exemple de copie, ou plagiat (minime, ici!) dans une expression non donnée comme citation. Le narrateur du Serviteur fidèle évoque «cette côte âpre où finissait la terre d'Occident, avec (...) ces villes mortes où pourrissent, à l'échauguette d'un château, les vieux canons de font à la gueule chargée de romarins, braqués à l'Anglais» (p. 63). C'est une réminiscence probable d'Au vieux Roscoff:

Va, ronfle au vent, vieux ronfleur,

Tiens toujours ta gueule enragée,

Braquée à l'Anglais!... et chargée

De maigre jonc-marin en fleur ${ }^{19}$.

«Et qu'il vente la peau du diable!» lance le narrateur du Naufrageur ${ }^{20}$, ce poème halluciné. Cette expression a rendu perplexes les commentateurs de Corbière. La lecture de La Prison maritime permet de l'éclairer. Il est question des bourrasques de la tempête:

(14) Jules Verne: texte et intertexte, Université de Haute-Bretagne, Rennes 2, 1987, p. 163.

(15) Les Amours jaunes, édité par Christian ANGELET, Paris, Le livre de poche, p. 238, v. 42.

(16) Ibid., pp. 234-236.
(17) Ibid., p. 222, vv. 7-8.

(18) Op. cit., p. 209.

(19) Op. cit., p. 225, vv. 39-42.

(20) Op. cit., p. 230, v. 20. 
Il existe une expression anglaise pour rendre l'impression que produit un vent de cette force: Il vente la peau du diable, et c'étaient en effet des lambeaux de peau glacée qui filaient dans la nuit, se plaquaient contre nos voiles, m'enveloppaient d'un suaire gluant (p. 268).

Plus loin, Hervé voit la voile «disparaître au-dessus des vagues comme un grand oiseau de mer, comme un lambeau de la peau du diable» (p. 272). Est-ce une expression tirée de Conrad, comme nous l'a aimablement écrit Michel Mohrt? Nous n'avons pas retrouvé l'expression originale dans les dictionnaires. Signalons que $\mathrm{La}$ Peau du diable est aussi le titre d'un roman maritime de Roger Vercel ${ }^{21}$.

On peut conjecturer la présence de «renvois implicites, ou allusions» aux Amours jaunes dans l'emploi que Michel Mohrt fait de technolectes certes propres au monde maritime, mais qui sont aussi très présents dans Gens de mer. La tradition de la quenouille figure même dans La Rapsode foraine, poème où Corbière décrit le pèlerinage de Saint-Anne-la-Palud et les invocations des fidèles à la Vierge:

Préserve notre cheminée

Des sorts et du monde malin...

A Pâques te sera donnée

Une quenouille avec du $\operatorname{lin}^{22}$.

Julienne, la mendiante pathétique des Moyens du bord, rappelle d'ailleurs la «rapsode» de Corbière. Après qu'on lui a fait l'aumône, «un sourire s'est creusé dans sa face ridée», note Michel Mohrt (p. 150). Le poète disait:

Tu verras dans sa face creuse

Se creuser, comme dans du bois,

Un sourire; et sa main galeuse

Te faire un vrai signe de croix ${ }^{23}$.

Le visage de Jean-Yves, rendu par la marée, est à moitié dévoré par les crabes. Sa fin rappelle celle du «Bossu Bitor», dans le poème éponyme ${ }^{24}$. Lady Cécilia, enfin, ressemble beaucoup au personnage-titre de L'Américaine, nouvelle maritime de Corbière. Il y dépeint un marin bourru et viril qui invite à son bord une jeune femme émancipée sensible au charme viril des matelots. Leur marivaudage rappelle d'autant plus celui de Lady Cécilia et d'Hervé que le narrateur, dans les deux récits, s'abandonne à une auto-dérision fort plaisante. Le recueil de Tristan Corbière fait donc sentir sa présence dans les romans maritimes de Michel Mohrt, et ce grâce à une complicité qui leur donne une couleur originale.

«Sans la liberté de blâmer, il n'est pas d'éloge flatteur»: telle est la devise du Figaro (empruntée, bien sûr, à Beaumarchais) où Michel Mohrt a écrit fort longtemps... Le style de l'auteur peut parfois susciter des réserves, surtout dans La Prison maritime. Il y use et abuse d'un procédé qui ne brille pas par sa légèreté, l'apostrophe au lecteur, parfois agrémentée d'une prolepse: «Certes, quand il connaîtra la suite des événements, le lecteur s'étonnera (...)» (p. 67), «Je préviens le lecteur (...) qu'il ne doit pas s'attendre à des développements scabreux (...)» (p. 86 ${ }^{25}$. Mais peut-être est-

(21) Paris, Albin Michel, 1952.

(22) Op. cit., p. 180, vv. 105-108.

(23) Ibid., p. 186, vv. 233-236.
(24) Ibid., p. 210.

(25) Cfr. aussi pp. 67, 96, 150, 152, 216, 222, 231, 234, 239, 248, 261... 
ce ici un tic propre au narrateur, Hervé, non à l'auteur, puisqu'il n'apparaît pas du tout dans les deux autres romans. La Prison maritime souffre peut-être d'une certaine surcharge rhétorique, dont témoignent encore telles prétéritions: «J'aimerais brosser un tableau du spectacle qui s'offrait à nos yeux, mais il y faudrait une plume moins malhabile que la miennes» ${ }^{26}$ (p. 53); «Parfois, au moment où le soleil s'enfonçait dans la mer au cours d'une agonie que je chercherais en vain à décrire (...)» (p. 82); «Je ne décrirai pas les spectacles classiques de la pauvreté irlandaise» (p. 153) (s'ensuivent alors la description de ces trois spectacles), ou telles prolepses (pp. 67, 96), ainsi que les morceaux de bravoure que sont les tableaux de la tempête. Cela-dit, ceux-ci sont, évidemment, les topoï obligés du récit maritime. Les portraits de Rose (p. 101) ou de Jennifer (p. 182), l'évocation du Boring Club (p. 219), sont un peu lourdement caricaturaux. Encore une fois, tout cela peut-être imputable au narrateur-scripteur, soucieux de «bien écrire». Mais Michel Mohrt nous livre aussi une vision hallucinée de Londres, qui est digne des Illuminations rimbaldiennes (p. 252); un bel éloge de l'exil et de sa «séduction amère» (p. 154); un plaidoyer pour le roman d'aventure, dont ce roman honore la tradition (p. 318). En prison, Hervé à l'ouïe exacerbée, et le passage où vibrent les moindres bruits du port est d'une richesse sensorielle qui rappelle Giono ou Colette (p. 301). L'humour jaillit souvent. Le marin «est un homme qui a un pot de peinture à la main» (p. 125). Hervé attribue au «non-conformisme» de Saint-Arthur le fait qu'il n'appartient pas à l'Académie française... Pas rancunière, cette dernière a couronné La Prison maritime en 1961, avant d'élire son auteur en son sein! Le propos est parfois rabelaisien:

Bravant la quarantaine, Olivier de Kersangar avait débarqué l'animal, le confiant pour la durée de notre séjour dans l'île à Lady Cécilia, devenue sa maîtresse officielle (je veux dire celle du chien) (p. 155).

Une scène burlesque montre les hommes d'équipage compissant le mât du navire, près duquel ils ont déposé un tas de sable, afin que le chien Du y fasse ses besoins, pour l'encourager à s'y mettre (p. 143)... L'humour noir apparaît dans Les Moyens du bord. Mme Le Cormier est morte après avoir acquis une «commodetombeau» (p. 170). L'humour blanc, pince-sans-rire, n'est pas absent. Pendant que Xavier, Martine et Ronan vivent leurs problèmes sentimentaux, le narrateur écrit: «Que se passait-il, pendant ce temps, à Lesguivy? Il ne se passait rien» (p. 147). Voilà une façon de faire un pied de nez au lecteur d'une fiction romanesque et à ses attentes. La chatte des époux Smallcat attrape un poisson et l'offre à ses petits (à ses «small cats»). «How extraordinary! dit Mrs Smallcat appuyée sur son mari. Would you do that for me, Albert?» (p. 107). Cela rappelle l'humour délicat de Saki. «Sachez attendre, prendre votre temps» (p. 183), dit Grace à Guillaume, qui s'inquiète du retard de Xavier. Mais Guillaume prend ce conseil pour une allusion à ses médiocres performances sexuelles, dans le lit-clos où ils se sont unis.

Michel Mohrt, enfin, nous renvoie souvent à une intertextualité qui sollicite nos compétences culturelles. Le cadavre de Tugdual, ballotté par la mer, «n'a plus de forme ni de nom dans aucune langue» (PM, p. 316). C'est une allusion au Sermon sur la mort, de Bossuet. Olivier «semblait mettre un point d'honneur à ne parler que de sujets techniques ou futiles en apparence: il m'a fallu du temps pour comprendre que là est la véritable élégance» (PM, p. 109). Ne dirait-on pas un portrait de Swann? Constatant que sa conception du théâtre est dépassée, Guillaume dit tristement: «C'est autre chose qu'il faudrait faire. Un théâtre d'ironie, de dérision...» (p. 195). 
C'est sans doute une allusion au théâtre de l'absurde, toujours populaire à l'époque où paraît Les Moyens du bord.

Bien que de droite, Michel Mohrt souligne la petitesse de Rine, et de la classe bourgeoise qu'elle sert, quand elle fait sentir à Louis «qu'il n'avait été admis à jouer avec ses petits maîtres que par un privilège extraordinaire» (SF, p. 60). Il mentionne aussi le fait que le breton est «proscrit des lycées et des écoles de l'Etat comme des collèges religieux et des petits séminaire» (PM, p. 46), ce qui s'inscrit en faux contre l'idée répandue que l'école privée l'a moins pourchassé que l'école laïque. C'est dire combien Michel Mohrt fuit le manichéisme.

Les techniques de narration, enfin, sont variées. C'est Hervé âgé qui raconte le récit des aventures qu'il a vécues jadis sur le Roi-Arthur. On a donc deux récits, celui du héros jeune et celui du narrateur qui reprend le fil de la narration à intervalles irréguliers: «Il y a un an, presque jour pour jour, que j’ai abandonné ce récit», écrit-il ainsi, au début du chapitre XVIII. Mais il n'y a qu'une page entre la fin du chapitre XVII et celui-ci: l'accélération est spectaculaire. Les analyses narratologiques peuvent donc se mener sur deux niveaux, ce qui est subtil. Dans Le Serviteur fidèle, le premier chapitre est vu du point de vue de Rine. Elle cède la place à un narrateur omniscient, mais l'avant-dernier chapitre est vu à travers le regard fruste de Lomic. Cela rappelle Le Bruit et la fureur, de Faulkner, où les chapitres ont aussi des points de focalisation différents. L'auteur fait ainsi varier l'expression, et accentue la dramatisation du récit. Le narrateur des Moyens du bord paraît neutre et omniscient, avant qu'il ne s'exprime soudain en son nom propre: «Et moi, chroniqueur de ces joies et de ces peines, de ces amours et de ces drames du temps des vacances, je songe qu'il me faudra bientôt terminer cette histoire d'un des derniers étés de l'avant-guerre» (p. 267). Le narrateur était en fait un témoin absent de l'anecdote, mais homodiégétique. Peut-être lui doiton ces rêveries sur Renan, Saint-Yves ou Tonquédec...

On espère donc avoir montré ici que Michel Mohrt est un écrivain qui mérite plus d'attention de la part des chercheurs, d'autant plus que son œuvre ne se limite pas aux trois romans que nous venons de présenter. Elle comprend des romans consacrés à la guerre, à l'Amérique, des essais, des traductions, ainsi que deux récits de souvenirs très émouvants, où la Bretagne occupe une place importante: La Maison du père ${ }^{27}$ et Vent d'Ouest ${ }^{28}$. Dans ce dernier volume, surtout, on rencontre les inspirateurs réels de certains personnages: la mendiante Julienne, le Grand Foc... ainsi que d'autres allusions précieuses aux traditions locales, à Tristan Corbière aussi. Mais, des trois romans abordés ici, c'est Les Moyens du bord qui nous enchante le plus. Les personnages sont attachants, fragiles et profonds à la fois. L'atmosphère un peu nonchalante de «grandes vacances», vécues avant la tourmente sous le regard vigilant de la mer invite à la nostalgie. L'écriture est simple, fraîche, iodée. Tout cela évoque le climat rayonnant d'un autre superbe roman de la Bretagne et de la mer, des tourments amoureux et de l'interrogation de l'homme face au destin qui l'attend: La Côte sauvage $^{29}$, de Jean-René Huguenin, dont Les Moyens du bord est comme un écho fraternel.

PASCAL RANNOU

(27) Gallimard, 1979.

(28) Olivier Orban, 1988. Les deux récits ont été publiés en un volume dans la collection Folio en
1990.

(29) Editions du Seuil, 1960. 